\title{
The need for quality self- management support in cancer care
}

\author{
Claire Foster
}

School of Health Sciences, University of Southampton, Southampton, UK

\section{Correspondence to} Prof Claire Foster, School of Health Sciences, University of Southampton, Southampton SO17 1BJ, UK:

C.L.Foster@soton.ac.uk

Accepted 8 June 2021

\section{Linked}

- http://dx.doi.org/10.1136/ bmjqs-2020-012051

\section{Check for updates}

(C) Author(s) (or their employer(s)) 2021. No commercial re-use. See rights and permissions. Published by BMJ.

To cite: Foster C. BMJ Qual Saf Epub ahead of print: [please include Day Month Year]. doi:10.1136/ bmjqs-2021-013366
The number of people living with and beyond cancer is rising rapidly. With earlier detection and better treatments many people are living for years following a diagnosis of cancer. Healthcare systems need to adapt to manage this demand and better meet the needs of this growing population. ${ }^{1}$

The consequences of cancer and its treatment are common, can arise at any point and may be long lasting. ${ }^{2}$ They can have a significant impact on daily life and include a range of physical symptoms and late effects such as pain, fatigue, bowel dysfunction; psychological concerns such as anxiety, depression and fear of recurrence; and social impacts including disruption to previously enjoyed activities, ability to work and relationships. ${ }^{3}$ Lifestyle changes and long-term medications to reduce the risk of recurrence, hospital appointments, routine surveillance and monitoring for signs of potential disease progression can create additional burden. People may lack confidence and struggle to manage the impact on their daily lives resulting in disrupted lives and futures. ${ }^{4}$ This can exacerbate mental health problems such as depression and anxiety. Low confidence to self-manage and depression have been associated with worse health and well-being outcomes up to 2 years after diagnosis. ${ }^{5}$ In addition, as most people are diagnosed with cancer in later life, many are also living with long-term conditions which can exacerbate problems further. ${ }^{6}$ Understanding how best to support people to manage the consequences of cancer and its treatment to reduce health crises and quality of life is a global concern. ${ }^{\text {? }}$

The good news is that appropriate support can reduce health crises, enhance confidence to manage and improve mental health, quality of life and other outcomes. ${ }^{89}$ The National Health Service (NHS) Long Term Plan ${ }^{10}$ states that by
2021 'where appropriate every person diagnosed with cancer will have access to personalized care, including needs assessment, a care plan, and health and wellbeing information and support' (p 61). Tailoring support and care according to need as soon as possible following diagnosis presents an opportunity to enhance confidence to manage consequences of cancer, mental health and quality of life outcomes during treatment. ${ }^{11}$ This support should continue beyond treatment because the consequences of treatment may persist over time, late effects may emerge which require selfmanagement and long-term lifestyle changes are usually required to reduce the risk of recurrence. New models of care are being developed and tested to support people after treatment and the evidence base is growing. ${ }^{12-14}$

Self-management is a term used to describe what people living with longterm conditions do to manage their condition. It includes 'the individual's ability to manage the symptoms, treatment, physical and psychosocial consequences and lifestyle changes inherent in living with a chronic condition' ( $\mathrm{p}$ 177). ${ }^{15}$ Self-management has more recently been applied in the context of cancer and additionally includes routine surveillance and self-monitoring for signs of disease progression to reduce the risk of recurrence. The benefits of successful self-management include reduced crises involving emergency admissions to hospital, improved quality of life and greater confidence to manage the impact of cancer on everyday life in spite of challenging consequences and the associated disruption. ${ }^{1617}$

While it is recognised that people need support to manage the wide-ranging impact on their everyday lives, selfmanagement support is inconsistently delivered in cancer care. ${ }^{7}$ A recent global 
call to action has highlighted the need for improved quality of self-management support to enable people to develop the knowledge, confidence and skills they need for optimal self-management. ${ }^{7}$ Without adequate self-management support, patients with cancer and survivors may struggle to manage the impact of cancer and treatment in their daily lives resulting in poorer health and well-being and poorer quality of survival. ${ }^{4}$

There is evidence that the right self-management support at the right time enables people to manage consequences of cancer during treatment and beyond..$^{812}{ }^{13}$ Howell et al ${ }^{18}$ demonstrate that both organisational and clinician barriers and enablers need to be considered in advance of implementation of self-management support to inform specific strategies for implementation within cancer care. The three cancer centres in Howell et al's ${ }^{18}$ study each identified barriers and enablers to implementation and readiness to change which require different approaches to support implementation. Howell et al ${ }^{18}$ highlight key considerations, based on their experience in ambulatory cancer care involving patients with colorectal, lung and lymphoma cancer, that need to be acknowledged and planned for prior to implementation of self-management support in cancer care. First, healthcare professionals and the organisations in which they work need support in shifting from a paternalistic culture to one that engages patients as partners in their care. Second, healthcare professionals and those in administration and management need to understand what self-management support is, what it involves and what the benefits could be for people living with and beyond cancer. Third, recognition that healthcare professionals need support, tools and skills to enhance their practice of self-management support.

Howell et al's ${ }^{18}$ study focuses on the preimplementation phase in a treatment setting. We can also learn from a UK study which assessed the introduction of supported self-management to the follow-up care pathway. The traditional approach to follow-up care where all patients have routine follow-up appointments and regular surveillance does not adequately address patients' needs and is not sustainable given the growing number of cancer survivors. ${ }^{19}$ The TrueNTH UK Supported Self-management and Follow-up Care Pathway (TrueNTH UK pathway) was introduced into parts of the NHS for men who have had primary prostate cancer treatment and are suitable for selfmanaged follow-up. ${ }^{20}$ The TrueNTH UK pathway includes a Band 4 support worker who is the key point of contact and support for men after treatment; a 4-hour workshop which provides information and supports the development of men's skills and confidence to self-manage; remote monitoring with hospital appointments only when test results indicate further investigation is necessary; access to test results through an online portal; and holistic needs assessment made through contact with the support worker. This new model of care has been shown to be more efficient and cost-effective than traditional follow-up and is acceptable to patients. ${ }^{12}$ It has also been adapted for patients with colorectal and breast cancer. A toolkit to support implementation of supported self-managed follow-up has been produced. ${ }^{20}$

The findings from Howell et al's ${ }^{18}$ study and the TrueNTH UK pathway align with a recent call to action from an international group of researchers and healthcare professionals-the Global Partners for Self-Management in Cancer-regarding workforce development and readying healthcare providers for successful self-management support. ${ }^{7}$ These include:

\section{A NECESSARY CULTURAL SHIFT}

Howell et $a l^{18}$ highlight the need for healthcare professionals and the organisations in which they work to shift from a paternalistic culture to a partnership arrangement where patients and healthcare professionals work together. This partnership will engage patients in their care and recovery to identify what matters to them, rather than what is the matter with them, and support them to be effective and confident self-managers equipped with the skills they need. ${ }^{78}$

To achieve this, Howell et al ${ }^{18}$ emphasise the need for healthcare professionals as well as healthcare organisation administrators and managers to be involved in the design of new self-management programmes. This will allow a full understanding of concerns, barriers and enablers for the implementation of self-management support. Subsequent implementation strategies will engage with professional and organisational understanding of what self-management support is, why it matters, and attempt to overcome barriers arising from this understanding.

Early barriers experienced in implementing the TrueNTH UK pathway included lack of buy-in from clinicians; concerns about patient safety; concern that health professionals would have limited contact with those patients doing well after treatment; concern that introduction of the pathway could result in reduced funding; lack of understanding of the components of the new pathway; challenges of having to navigate multiple administrative systems; and challenges implementing and integrating the information technology (IT) system. These barriers were identified in the early stages of implementation and where these challenges were overcome, the pathway was successfully introduced, was acceptable to patients and reduced costs. ${ }^{12}$ Howell et al's study suggests that these barriers, both in terms of healthcare professional and organisational readiness to change, could have been identified at an earlier stage and strategies developed to inform smoother uptake prior to implementation.

Enablers to support the cultural shift needed to implement the TrueNTH UK pathway included identifying clinical leads to support early engagement with a wide range of stakeholders including commissioners, 
clinical governance and service improvement leads, IT to support integration of self-management support into digital systems and administrative support to overcome the complexity brought about by multiple administrative systems. A working group led by a healthcare professional (eg, urologist, oncologist, clinical nurse specialist) responsible for implementation of the TrueNTH UK pathway and taking decisions on the development of clinical guidelines and governance protocols supported the implementation and delivery of safe and effective follow-up care. This group also supported teams to maximise understanding of the new pathway and what it entails, as well as collection of data to support accurate cost modelling.

\section{PREPARING THE WORKFORCE}

Howell et $a l^{18}$ emphasise that for successful implementation, a common understanding of what selfmanagement in cancer care means, why it is important and what good self-management support looks like is a necessary starting point. Healthcare professionals need support to acquire the knowledge and skills required to enable patients to self-manage effectively and agree core curricula to support this.

TrueNTH UK also emphasised the importance of wide stakeholder engagement to identify concerns and needs of different stakeholders throughout this process to develop a common understanding and identify local need and encourage buy-in. A local clinical champion to help drive this forward is beneficial to support clinical teams responsible for implementation to understand what is required and involve them in early discussions to shape the pathways, guidance and associated protocols. ${ }^{20}$

\section{CORE SET OF OUTCOME MEASURES}

Both Howell et $a l^{18}$ and TrueNTH UK highlighted the importance of data to demonstrate the benefits of supported self-management, such as reduced emergency admissions. In order to determine whether or not self-management support is effective, consensus as to desired outcomes also needs to be established. An agreed core set of patient-reported outcome measures is also required. Evidence suggests that self-efficacy to manage the consequences of cancer and its treatment should be included as one indicator of successful self-management as it is both amenable to intervention and is a key predictor of health and well-being outcomes. 521

\section{EXPAND REACH AND ACCESS TO SELF- MANAGEMENT SUPPORT}

Both SMARTCare (Howell et al ${ }^{18}$ ) and TrueNTH UK pathway are designed to form part of routine care. The TrueNTH UK pathway has been integrated into routine care and is therefore not an optional extra: all eligible patients are placed on the pathway. Patients are supported to understand what self-management support is and how to engage with it. In contrast, optional programmes tend to attract well-educated patients who already self-manage well. ${ }^{22}$ Consequently, those implementing self-management support need to consider the diversity of need within the population served so that those with greatest need are the focus of attention. The needs of the population served need to be assessed with involvement of those typically under-represented. Meaningful patient engagement to uncover needs should feed into the development of self-management support. Self-management support needs to come in a number of forms including workshops, digital support and one-to-one clinical conversations that are culturally sensitive and inclusive of those with low health literacy.

\section{CONCLUSION}

For self-management support to be a success, we need to anticipate, uncover and overcome organisational and healthcare professional barriers to implementation, have a clear understanding of what good looks like, have agreement regarding how to evidence successful self-management support and gather evidence of good practice so that this can be shared, replicated and adapted as appropriate. Champions in organisations across the globe are needed to lead the way in high-quality and consistent self-management support to improve the lives of those living with and beyond cancer.

Funding The authors have not declared a specific grant for this research from any funding agency in the public, commercial or not-for-profit sectors.

Competing interests None declared.

Patient consent for publication Not required.

Provenance and peer review Commissioned; internally peer reviewed.

\section{REFERENCES}

1 Rowland JH, Hewitt M, Ganz PA. Cancer survivorship: a new challenge in delivering quality cancer care. J Clin Oncol 2006;24:5101-4.

2 Foster C, Wright D, Hill H, et al. Psychosocial implications of living 5 years or more following a cancer diagnosis: a systematic review of the research evidence. Eur J Cancer Care 2009;18:223-47.

3 Hewitt MGS, Stovall E. From cancer patient to cancer survivor: lost in transition. Washington, DC: National Academies Press, 2006.

4 Foster C, Fenlon D. Recovery and self-management support following primary cancer treatment. Br J Cancer 2011;105 Suppl 1:S21-8.

5 Foster C, Haviland J, Winter J, et al. Pre-Surgery depression and confidence to manage problems predict recovery trajectories of health and wellbeing in the first two years following colorectal cancer: results from the crew cohort study. PLoS One 2016;11:e0155434.

6 Cummings A, Grimmett C, Calman L, et al. Comorbidities are associated with poorer quality of life and functioning and worse symptoms in the 5 years following colorectal cancer 
surgery: Results from the ColoREctal Well-being (CREW) cohort study. Psychooncology 2018;27:2427-35.

7 Howell D, Mayer DK, Fielding R, et al. Management of cancer and health after the clinic visit: a call to action for selfmanagement in cancer care. J Natl Cancer Inst 2020.

8 Howell DD. Supported self-management for cancer survivors to address long-term biopsychosocial consequences of cancer and treatment to optimize living well. Curr Opin Support Palliat Care 2018;12:92-9.

9 Foster C, Grimmett C, May CM, et al. A web-based intervention (restore) to support self-management of cancerrelated fatigue following primary cancer treatment: a multicentre proof of concept randomised controlled trial. Support Care Cancer 2016;24:2445-53.

10 Service NH. The NHS long term plan. National Health Service, 2019.

11 Carlson LE, Waller A, Mitchell AJ. Screening for distress and unmet needs in patients with cancer: review and recommendations. J Clin Oncol 2012;30:1160-77.

12 Frankland J, Brodie H, Cooke D, et al. Follow-Up care after treatment for prostate cancer: evaluation of a supported selfmanagement and remote surveillance programme. BMC Cancer 2019;19:368.

13 van der Hout A, van Uden-Kraan CF, Holtmaat K, et al. Role of eHealth application Oncokompas in supporting selfmanagement of symptoms and health-related quality of life in cancer survivors: a randomised, controlled trial. Lancet Oncol 2020;21:80-94.

14 Hernandez Silva E, Lawler S, Langbecker D. The effectiveness of mHealth for self-management in improving pain, psychological distress, fatigue, and sleep in cancer survivors: a systematic review. J Cancer Surviv 2019;13:97-107.
15 Barlow J, Wright C, Sheasby J, et al. Self-Management approaches for people with chronic conditions: a review. Patient Educ Couns 2002;48:177-87.

16 Budhwani S, Wodchis WP, Zimmermann C, et al. SelfManagement, self-management support needs and interventions in advanced cancer: a scoping review. BMJ Support Palliat Care 2019;9:12-25.

17 Haase KR, Sattar S, Hall S, et al. Systematic review of self-management interventions for older adults with cancer. Psychooncology 2021. doi:10.1002/pon.5649. [Epub ahead of print: 05 Feb 2021].

18 Howell D, Powis M, Kirkby R, et al. Improving the quality of self-management support in ambulatory cancer care: a mixed-method study of organisational and clinician readiness, barriers and enablers for tailoring of implementation strategies to multisites. BMJ Qual Saf 2021. doi:10.1136/bmjqs-2020-012051. [Epub ahead of print: 16 Mar 2021].

19 Armes J, Crowe M, Colbourne L, et al. Patients' supportive care needs beyond the end of cancer treatment: a prospective, longitudinal survey. J Clin Oncol 2009;27:6172-9.

20 UK PC. Personalised stratified follow-up. Available: https:/ prostatecanceruk.org/for-health-professionals/truenthsupported-self-management 2016

21 Foster C, Grimmett C, May CM, et al. A web-based intervention (restore) to support self-management of cancerrelated fatigue following primary cancer treatment: a multicentre proof of concept randomised controlled trial. Support Care Cancer 2016;24:2445-53.

22 Wilson PM. The UK expert patients program: lessons learned and implications for cancer survivors' self-care support programs. J Cancer Surviv 2008;2:45-52. 